\title{
Distribuição espacial de aves em um fragmento florestal do campus da Universidade Estadual de Londrina, Norte do Paraná, Brasil
}

\author{
Márcio Rodrigo Gimenes ${ }^{1}$ \\ Luiz dos Anjos ${ }^{2}$
}

\begin{abstract}
Spatial distribution of birds in a forest fragment at Londrina State University campus, Northern Paraná State, Brazil. Spatial distribution of birds was studied in 10 ha forest fragment at Londrina State University, southern Brazil (23ำ 'S, $\left.51^{\circ} 10^{\prime} \mathrm{W}\right)$. Five habitats were categorized in the forest fragment: four related to the edges and one related to the forest interior. A line transect, crossing all these habitats, was used to identify the bird species one day each two weeks from September 1996 to September 1997 totaling 57 days of samplings. The forest was divided into three levels: understory (bellow $3 \mathrm{~m}$ ), mid-levels (3-7 m) and canopy (above $7 \mathrm{~m}$ ). Occurrence frequencies in each habitat and each forest level were calculated for each of the 62 species identified during the field work. The number of species in each level was similar (43 species in the understory, 42 at mid-levels and 40 in the canopy). Edges had higher number of species $(51,46,33$ and 30 species) and most of them with more than $50 \%$ of occurrence frequency. By contrast, forest had the lowest number of bird species ( 29 species), $80 \%$ of them with less than $50 \%$ of occurrence frequency, what suggest that the edge effect is strong on this forest fragment.

KEY WORDS. Spatial distribution, birds, forest fragment, northern Paraná State, south of Brazil
\end{abstract}

Densas florestas cobriam quase todo o estado do Paraná no início deste século (MAACK 1981). Devido ao intenso processo de uso da terra, especialmente na região norte, resta atualmente menos de $8 \%$ da área original de floresta do Estado (sos FUNDAÇÃo MATA ATLÂNTICA \& INPE 1990). No norte do Paraná a paisagem mais comum é composta por grandes áreas de agricultura ou de criação de gado; pequenos e isolados fragmentos florestais, normalmente menores que 50 ha, são encontrados em algumas regiões.

Muitas das espécies de aves que primitivamente viviam em florestas contínuas não conseguem sobreviver em tais fragmentos. Vários autores mencionam um decréscimo no número de espécies de aves com a diminuição da área da floresta (e.g. WiLlis 1979; BIERREGAARD \& LOVEJOY 1989). Além da área reduzida, situação que diminui drasticamente a disponibilidade de recursos alimentares, e do

1) Curso de Pós-Graduação em Ecologia de Ecossistemas Aquáticos Continentais, Universidade Estadual de Maringá. Avenida Colombo 3690, Bloco G-90, 87020-900 Maringá, Paraná, Brasil.

2) Departamento de Biologia Animal e Vegetal, Universidade Estadual de Londrina. Caixa Postal 6001, 86051-970 Londrina, Paraná, Brasil. Bolsista do CNPq. 
isolamento, as populações de aves florestais que conseguem viver nestes fragmentos sofrem pressão ecológica contínua das condições bióticas e abióticas do ambiente aberto em redor, especialmente na borda da floresta, o que denomina-se efeito de borda (YAHNER 1988). O efeito de borda tende a ser maior e mais prejudicial à sobrevivência de organismos florestais quanto menor for o fragmento (FORMAN et al. 1976; GALLI et al. 1976).

Muitos trabalhos que abordam os efeitos da fragmentação florestal sobre comunidades de aves foram conduzidos nas regiões temperadas (e.g. MOORE \& HOOPER 1975; HOLMES 1990a,b; WilCOVE \& ROBINSON 1990; BLONDEL 1991), mas são poucos os realizados no sul e sudeste do Brasil, destacando-se os de WILLIS (1979), ANJOS \& LAROCA (1989), ALEIXo \& Vielliard (1995) e ANJOS \& BoçON (1999).

O objetivo deste trabalho é analisar a distribuição espacial e a composição da comunidade de aves em um fragmento florestal e em seu entorno no norte do Estado do Paraná.

\section{MATERIAL E MÉTODOS}

\section{Local de estudo}

O fragmento florestal estudado ocupa uma área de aproximadamente 10 ha do campus da Universidade Estadual de Londrina ( $\left.23^{\circ} 15^{\prime} \mathrm{S}, 51^{\circ} 10^{\prime} \mathrm{W}\right)$. A floresta que compõe este fragmento, estacional semidecidual, sofreu ação antrópica no passado mas já readquiriu uma fitofisionomia similar à natural. No fragmento são encontradas várias espécies arbóreas, sendo as famílias mais comuns Euphorbiaceae, Moraceae, Leguminosae, Lauraceae e Meliaceae. A vegetação arbustiva é bastante abundante.

\section{Métodos}

Cinco ambientes foram reconhecidos e codificados com letras neste fragmento, principalmente em função do seu entorno: borda de floresta composta por gramado e árvores esparsas (A); borda de floresta composta por gramíneas altas (B); borda de floresta composta por plantação de milho (C); borda de floresta composta por capoeira (D) e interior de floresta (E). Um transecto foi determinado de modo a amostrar todos estes ambientes sempre na seqüência A-B-C-D-E (Fig. 1).

Este transecto foi percorrido, semanalmente, de setembro de 1996 a setembro de 1997, totalizando 57 dias de amostragens. Ao longo do transecto o observador (sempre o mesmo) identificava cada ave visualizada, narrando em um gravador cassete portátil a espécie, o ambiente e o estrato em que foi observada. Três estratos foram considerados: inferior (até $3 \mathrm{~m}$ ), médio (entre 3 e $7 \mathrm{~m}$ ) e superior (acima de $7 \mathrm{~m}$ ). Cada espécie era registrada somente uma vez em cada ambiente e em cada estrato em um mesmo dia de amostragem. O percurso do transecto era iniciado sempre uma hora após o sol nascente. O tempo de percurso do transecto era sempre o mesmo ( 3 horas) sendo que o observador procurava manter a mesma velocidade durante todo o trajeto. A identificação e a nomenclatura das espécies foi baseada em MEYER DE SCHAUENSEE (1982) e atualizada segundo SCHERER-NETO \& STRAU$\mathrm{BE}$ (1995). 


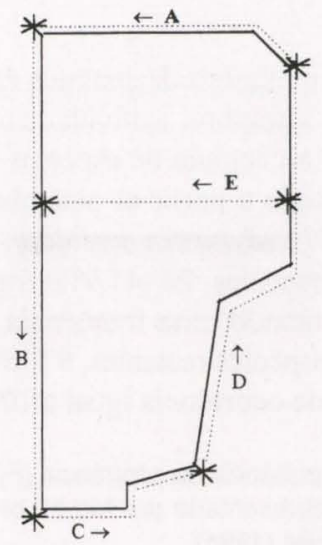

Fig. 1. Transecto determinado no fragmento estudado (10 ha) cruzando os ambientes $A, B$, C, D e E.

A partir dos dados coletados ao longo dos 57 dias de amostragens foram calculadas as freqüências de ocorrências das espécies de aves (número de amostras em que a espécie esteve presente dividido por 57 e multiplicado por 100) e a repartição delas nos ambientes e nos estratos (número de amostragens em que a espécie esteve presente em determinado ambiente ou estrato, dividido pelo total de dias de amostragem em que ela esteve presente e multiplicado por 100). Chi-quadrado foi usado para determinar se há diferença significativa entre os ambientes ou entre os estratos frequentados por uma espécie. A similaridade, em relação à composição das espécies, entre os ambientes, foi avaliada através do índice de Simpson.

\section{RESULTADOS E DISCUSSÃO}

Um total de 62 espécies de aves foi registrado no transecto (Tab. I).

Pequenos fragmentos florestais em bom estado de conservação, mas com forte alteração do entorno, foram estudados por WiLLIS (1979), que registrou 93 espécies de aves em um fragmento de floresta subtropical de 21 ha na região de Campinas, e por ANJOS (1990), que registrou 88 espécies de aves em 3,6 ha de um fragmento natural de floresta de araucária em Curitiba. O número obtido no Campus da Universidade Estadual de Londrina foi inferior a estes dois locais possivelmente pela menor área, em relação ao de Campinas, e pelo estado mais precário de conservação, em relação ao de Curitiba. No maior e melhor conservado fragmento florestal encontrado no norte do Paraná, o Parque Estadual Mata dos Godoy (656 ha), foram registradas 288 espécies de aves (ANJOS et al. 1997).

O número médio (média aritmética) de espécies foi um pouco maior (29 espécies) nos meses mais quentes do ano (setembro a dezembro), quando as médias de temperatura em Londrina estão entre $20 \mathrm{e} 23,7^{\circ} \mathrm{C}$ e menor (24 espécies) nos meses mais frios (junho a agosto), quando as médias estão entre 16,8 e $19,1^{\circ} \mathrm{C}$ (MAACK 1981). Possivelmente a maior atividade das aves no período reprodutivo (que inicia em setembro para a maioria das espécies) facilitou a visualização contribuindo para a identificação de um maior número de espécies. VIELLIARD \& SiLVA (1990) 
relacionaram um aumento no número de contatos em uma comunidade de aves de Lençóis Paulista, a partir de setembro, à atividade reprodutiva das espécies. Outro aspecto a ser considerado é a chegada de espécies reconhecidamente migratórias (SICK 1997). Na área de estudo a partir de setembro foram registradas Tyrannus savana (Linnaeus, 1758) e Myiodynastes maculatus (Müller, 1776).

Dentre o total de 62 espécies, 26 (41,9\%) foram registradas em menos de 8 dias de amostragens apresentando uma freqüência de ocorrência geral inferior a $14 \%$ (Tab. I). Dentre as 36 espécies restantes, 9 (15\%) foram registradas em todas as amostragens (freqüência de ocorrência igual a 100\%).

Tabela I. Registros mensais e freqüência de ocorrência (FO) das espécies de aves, apresentadas seguindo nomenclatura apresentada por MEYER DE SCHAUENSEE (1982) e atualizada segundo SCHERER-NETO \& STRAUBE (1995).

\begin{tabular}{|c|c|c|c|c|c|c|c|c|c|c|c|c|c|c|}
\hline \multirow{2}{*}{ Familia } & \multirow{2}{*}{ Espécie } & \multicolumn{12}{|c|}{ Meses } & \multirow{2}{*}{$\begin{array}{l}\text { FO } \\
(\%)\end{array}$} \\
\hline & & J & $\mathrm{F}$ & M & A & M & J & $\mathrm{J}$ & A & $\mathrm{S}$ & 0 & $\mathrm{~N}$ & D & \\
\hline Cathartidae & Coragyps atratus (Bonaparte, 1793) & & $x$ & & & $x$ & $x$ & $x$ & $x$ & $x$ & & & $x$ & 14 \\
\hline Accipitridae & Buteo magnirostris (Gmelin, 1788) & $x$ & $x$ & $x$ & $\mathrm{x}$ & $x$ & $x$ & $x$ & $x$ & $x$ & $x$ & $x$ & $\mathrm{x}$ & 77 \\
\hline Charadriidae & Vanellus chilensis (Gmelin 1789) & $x$ & & & $x$ & $x$ & & $x$ & $x$ & $x$ & $x$ & $x$ & & 23 \\
\hline \multirow[t]{7}{*}{ Columbidae } & Columba picazuro Temminck, 1813 & $x$ & $x$ & $\mathrm{x}$ & $\mathrm{x}$ & $x$ & $\mathrm{x}$ & $x$ & $x$ & $x$ & $x$ & $x$ & $\mathrm{x}$ & 53 \\
\hline & Columba cayennensis Bonnaterre, 1792 & $x$ & $x$ & & $\mathrm{x}$ & $x$ & $x$ & & $x$ & & $x$ & $x$ & $\mathrm{x}$ & 24 \\
\hline & Zenaida auriculata Chubb, 1819 & $x$ & $x$ & $x$ & $\mathrm{x}$ & $x$ & $x$ & $x$ & $\mathrm{x}$ & $\mathrm{x}$ & $x$ & $x$ & $\mathrm{x}$ & 67 \\
\hline & Columbina picui (Temminck, 1813) & & & & & & & & & $x$ & & & $x$ & 3 \\
\hline & Columbina talpacoti Temminck, 1811 & $x$ & $x$ & $x$ & $\mathrm{x}$ & $x$ & & & $x$ & $\mathrm{x}$ & $x$ & $x$ & $\mathrm{x}$ & 38 \\
\hline & Leptotila verreauxi Bonaparte 1855 & & & & & & & $x$ & & & & & & 2 \\
\hline & Leptotila rufaxilla Richard \& Bernard, 1712 & & & & $\mathrm{x}$ & $x$ & & & $x$ & & & & & 7 \\
\hline \multirow[t]{3}{*}{ Psittacidae } & Aratinga leucophthalmus (Mâler, 1776) & $\mathrm{x}$ & & & & & $\mathrm{x}$ & & & & & & $x$ & 7 \\
\hline & Pyrrhura frontalis (Vieillot, 1817) & & & & $x$ & $x$ & & & & & & & & 3 \\
\hline & Pionus maximiliani (Kuhl, 1820) & & & $x$ & & & $\mathrm{x}$ & & & & & & & 3 \\
\hline \multirow[t]{3}{*}{ Cuculidae } & Piaya cayana (Linnaeus, 1766) & $x$ & $x$ & $x$ & $x$ & $x$ & $x$ & $x$ & $x$ & $x$ & $x$ & $x$ & $x$ & 61 \\
\hline & Crotophaga ani (Linnaeus, 1758) & $x$ & $x$ & $x$ & $\mathrm{x}$ & $x$ & $x$ & $x$ & $x$ & $\mathrm{x}$ & $x$ & $x$ & $\mathrm{x}$ & 63 \\
\hline & Guira guira Gmelin, 1831 & $x$ & $x$ & $x$ & $x$ & & $x$ & $x$ & $x$ & $x$ & $x$ & $x$ & $x$ & 31 \\
\hline Caprimulgidae & Nyctidromus albicollis (Gmelin, 1789) & & & & & & & & & & & & $\mathrm{x}$ & 3 \\
\hline \multirow[t]{3}{*}{ Trochilidae } & Phaethornis eurynome (Lesson, 1832) & $x$ & & $x$ & $\mathrm{x}$ & & & & & $x$ & $x$ & $x$ & $x$ & 24 \\
\hline & Phaethornis pretrei (Lesson \& Delattre, 1839) & & & & $\mathrm{x}$ & $x$ & $\mathrm{x}$ & & & & & & & 5 \\
\hline & Melanotrochilus fuscus (Vieillot, 1817) & $x$ & $x$ & & $\mathrm{x}$ & $x$ & $\mathrm{x}$ & & & & & & $x$ & 24 \\
\hline Bucconidae & Nonnula rubecula (Spix, 1824) & & & & & & & & & & & & $x$ & 2 \\
\hline \multirow[t]{3}{*}{ Picidae } & Picumnus temminckii Temminck, 1825 & & & & $x$ & $x$ & $x$ & & & & $x$ & & $x$ & 16 \\
\hline & Veniliornis spilogaster (Wagler, 1827) & & & $x$ & & & $x$ & & & & & $\mathrm{x}$ & $x$ & 9 \\
\hline & Dryocopus lineatus (Linnaeus, 1766) & & & & $x$ & & $x$ & & $x$ & & & & & 5 \\
\hline \multirow[t]{2}{*}{ Furnariidae } & Furnarius rufus (Gmelin, 1816) & & & & & $x$ & & & & $x$ & & & & 3 \\
\hline & Synallaxis ruficapilla Vieillot 1819 & $x$ & $x$ & & $\mathrm{x}$ & $x$ & $x$ & $x$ & $x$ & $x$ & $x$ & $x$ & $x$ & 60 \\
\hline \multirow{4}{*}{ Formicariidae } & Thamnophilus caerulescens Vieillot, 1816 & $\mathrm{x}$ & $x$ & $\mathrm{x}$ & $x$ & $x$ & $x$ & $x$ & $x$ & $x$ & $x$ & $x$ & $\mathrm{x}$ & 82 \\
\hline & Dysithamnus mentalis (Temminck, 1823) & $x$ & $\mathrm{x}$ & $x$ & $\mathrm{x}$ & $x$ & $x$ & $x$ & $x$ & & $x$ & $\mathrm{x}$ & $\mathrm{x}$ & 33 \\
\hline & Pyriglena leucoptera (Vieillot, 1818) & & & & & $x$ & $\mathrm{x}$ & & & & & & & 5 \\
\hline & Conopophaga lineata (Wied, 1831) & & & & $\mathrm{x}$ & $x$ & & $x$ & & & & & & 5 \\
\hline \multirow[t]{11}{*}{ Tyrannidae } & Leptopogon amaurocephalus (Tschud, 1846) & & $x$ & & $\mathrm{x}$ & $x$ & $x$ & $x$ & $x$ & & $x$ & $\mathrm{x}$ & $x$ & 37 \\
\hline & Myiornis auricularis (Vieillot, 1818) & & & & $\mathrm{x}$ & & $x$ & & & $x$ & & & & 5 \\
\hline & Lathrotriccus euleri (Cabanis, 1868) & & $x$ & & $x$ & $x$ & $x$ & & & & & & $x$ & 10 \\
\hline & Colonia colonus (Vieillot, 1818) & & & & & & $\hat{x}$ & & & & & & & 2 \\
\hline & Machetornis rixosus (Vieillot, 1819) & $x$ & $x$ & & & $x$ & $x$ & $x$ & $x$ & & & & $\mathrm{x}$ & 30 \\
\hline & Tyrannus savana (Linnaeus, 1758) & $x$ & $x$ & & & & & & & $x$ & $x$ & $x$ & $\mathrm{x}$ & 30 \\
\hline & Tyrannus melancholichus (Vieillot, 1819) & & & $x$ & & & & & & & & & & 2 \\
\hline & Megarhynchus pitangua (Linnaeus, 1766) & $x$ & $\mathrm{x}$ & $x$ & $\mathrm{x}$ & & $x$ & $x$ & & $x$ & $x$ & $x$ & $x$ & 31 \\
\hline & Myiodynastes maculatus (Moller, 1776) & & $x$ & & & & & & & $x$ & $x$ & $x$ & $x$ & 24 \\
\hline & Myiozetetes similis (Spix, 1825) & $\mathrm{x}$ & & & & & & & & & & & & 2 \\
\hline & Pitangus sulphuratus (Linnaeus, 1766) & $x$ & $\mathrm{x}$ & $x$ & $x$ & $x$ & $x$ & $x$ & $x$ & $x$ & $x$ & $x$ & $x$ & 91 \\
\hline
\end{tabular}


Tabela I. Continuação.

\begin{tabular}{|c|c|c|c|c|c|c|c|c|c|c|c|c|c|c|}
\hline \multirow{2}{*}{ Familia } & \multirow{2}{*}{ Espécie } & \multicolumn{12}{|c|}{ Meses } & \multirow{2}{*}{$\begin{array}{l}\text { FO } \\
(\%)\end{array}$} \\
\hline & & $J$ & $\mathrm{~F}$ & M & A & M & $\mathrm{J}$ & $J$ & A & $\mathrm{s}$ & 0 & $\mathrm{~N}$ & D & \\
\hline Troglodytidae & Troglodytes aedon Vieillot, 1807 & $x$ & & $\mathrm{x}$ & $x$ & $x$ & & & $x$ & $x$ & $x$ & $x$ & $x$ & 31 \\
\hline \multirow[t]{3}{*}{ Turdidae } & Turdus rufiventris (Vieillot, 1818) & $x$ & $x$ & $\mathrm{x}$ & $\mathrm{x}$ & $x$ & $\mathrm{x}$ & $x$ & $\mathrm{x}$ & $\mathrm{x}$ & $x$ & $\mathrm{x}$ & $\mathrm{x}$ & 53 \\
\hline & Turdus leucomelas (Vieillot, 1818) & $x$ & $x$ & $x$ & $x$ & $x$ & $x$ & $x$ & $\mathrm{x}$ & $x$ & $x$ & $\mathrm{x}$ & $x$ & 72 \\
\hline & Turdus amaurochalinus Cabanis, 1851 & $\mathrm{x}$ & $\mathrm{x}$ & $\mathrm{x}$ & $\mathrm{x}$ & $\mathrm{x}$ & $x$ & & & & $\mathrm{x}$ & $\mathrm{x}$ & $\mathrm{x}$ & 31 \\
\hline \multirow[t]{10}{*}{ Emberizidae } & Zonotrichia capensis (Müller, 1776) & & $\mathrm{x}$ & $\mathrm{x}$ & $x$ & $x$ & $x$ & & & $\mathrm{x}$ & $\mathrm{x}$ & $\mathrm{x}$ & $\mathrm{x}$ & 23 \\
\hline & Volatinia jacarina (Linnaeus, 1758) & & $\mathrm{x}$ & $\mathrm{x}$ & $x$ & $\mathrm{x}$ & $\mathrm{x}$ & & & & & & & 33 \\
\hline & Sporophila lineola (Linnaeus, 1758) & & & & & & & & & $\mathrm{x}$ & & & & 2 \\
\hline & Sporophila caerulescens (Vieillot, 1817) & & & & & & & & & & & & $x$ & 2 \\
\hline & Arremon flavirostris (Swainson, 1837) & $\mathrm{x}$ & & $\mathrm{x}$ & & & $\mathrm{x}$ & & & & & & $\mathrm{x}$ & 12 \\
\hline & Saltator similis (Lafresnaye \& d'Orbigny, 1837) & $x$ & $x$ & $x$ & $x$ & $\mathrm{x}$ & $x$ & $\mathrm{x}$ & & & & $\mathrm{x}$ & $x$ & 24 \\
\hline & Tachyphonus coronatus (Vieillot, 1822) & $\mathrm{x}$ & $\mathrm{x}$ & & $x$ & $\mathrm{x}$ & $x$ & & & & & $\mathrm{x}$ & $x$ & 24 \\
\hline & Thraupis sayaca (Linnaeus, 1766) & $x$ & $x$ & & $x$ & $\mathrm{x}$ & $x$ & $\mathrm{x}$ & $\mathrm{x}$ & & $\mathrm{x}$ & $\mathrm{x}$ & $\mathrm{x}$ & 42 \\
\hline & Euphonia chlorotica (Linnaeus, 1766) & & & & & $\mathrm{x}$ & & & & & & & & 2 \\
\hline & Trichothraupis melanops (Vieillot, 1818) & & & $\mathrm{x}$ & $x$ & & & & & & & & & 7 \\
\hline \multirow[t]{4}{*}{ Parulidae } & Parula pitiayumi (Vieillot, 1817) & $x$ & $\mathrm{x}$ & & $x$ & $x$ & $x$ & & $\mathrm{x}$ & & $\mathrm{x}$ & $\mathrm{x}$ & $\mathrm{x}$ & 30 \\
\hline & Basileuterus culicivorus (Lichtenstein, 1830) & $x$ & $\mathrm{x}$ & $\mathrm{x}$ & $x$ & $\mathrm{x}$ & $x$ & $\mathrm{x}$ & $\mathrm{x}$ & & $\mathrm{x}$ & $\mathrm{x}$ & $x$ & 60 \\
\hline & Basileuterus leucoblepharus (Vieillot, 1817) & & & & $x$ & $x$ & & & & & & & & 3 \\
\hline & Conirostrum speciosum (Temminck, 1824) & $\mathrm{x}$ & & & $x$ & & $x$ & & $\mathrm{x}$ & & & $\mathrm{x}$ & $x$ & 23 \\
\hline Vireonidae & Cyclarhis gujanensis (Gmelin, 1789) & & & & & $x$ & $x$ & & & & & & & 5 \\
\hline Icteridae & Molothrus bonariensis (Gmelin, 1789) & & & $x$ & $x$ & $\mathrm{x}$ & $x$ & $\mathrm{x}$ & & $\mathrm{x}$ & $\mathrm{x}$ & $\mathrm{x}$ & $x$ & 33 \\
\hline Ploceidae & Passer domesticus (Linnaeus, 1758) & $\mathrm{x}$ & $x$ & & $x$ & $\mathrm{x}$ & $x$ & $x$ & & & $x$ & $x$ & $x$ & 17 \\
\hline
\end{tabular}

Muitas das espécies com baixa freqüência de ocorrência vivem em áreas abertas, visitando, irregularmente, o fragmento como é o caso de Vanellus chilensis (Gmelin, 1789), Guira guira (Gmelin, 1831), Tyrannus savana e Sporophila caerulescens (Vieillot, 1817). Entretanto, outras são florestais, sendo que a baixa freqüência de ocorrência pode estar relacionada à baixa população no fragmento como no caso de Conopophaga lineata (Wied, 1831), Basileuterus leucoblepharus (Vieillot, 1817) e Trichothraupis melanops (Vieillot, 1818). Um grupo de aves florestais particularmente raro em fragmentos é o dos Psittacidae que estiveram representados por pequeno número de espécies e com baixa freqüência de ocorrência (3-7\%): Aratinga leucophthalmus (Müller, 1776), Pyrrhura frontalis (Vieillot, 1817) e Pionus maximiliani (Kuhl, 1820). WiLLIS (1979) menciona que grandes frugívoros como os Psittacidae são rapidamente extintos em pequenos fragmentos por necessitarem de diferentes espécies de árvores frutificando em diferentes estações do ano. As três espécies de Psittacidae certamente não vivem apenas no fragmento mas o exploram em diferentes períodos do ano para aproveitar determinados recursos.

$\mathrm{O}$ ambiente mais rico em número de espécies foi o $\mathrm{B}$ (51 espécies) seguido pelos ambientes D, A, C e E (46, 33, 30 e 29 espécies respectivamente, Tab. I). O ambiente B apresentou também a maior percentagem de espécies com freqüência de ocorrência superior a $50 \%$ (39,2\%) seguido pelo ambiente D (34,8\%, Fig. 2). Por outro lado os ambientes A, C e E apresentaram baixa percentagem de espécies com freqüência de ocorrência superior a 50\% $(9,1 \% ; 13,3 \%$ e $20,7 \%$ respectivamente).

Os dados acima sugerem que entornos de fragmentos florestais compostos por gramíneas altas (ambiente B) e capoeira (ambiente D) contribuem para uma maior diversidade de espécies de aves e para uma maior regularidade na presença 


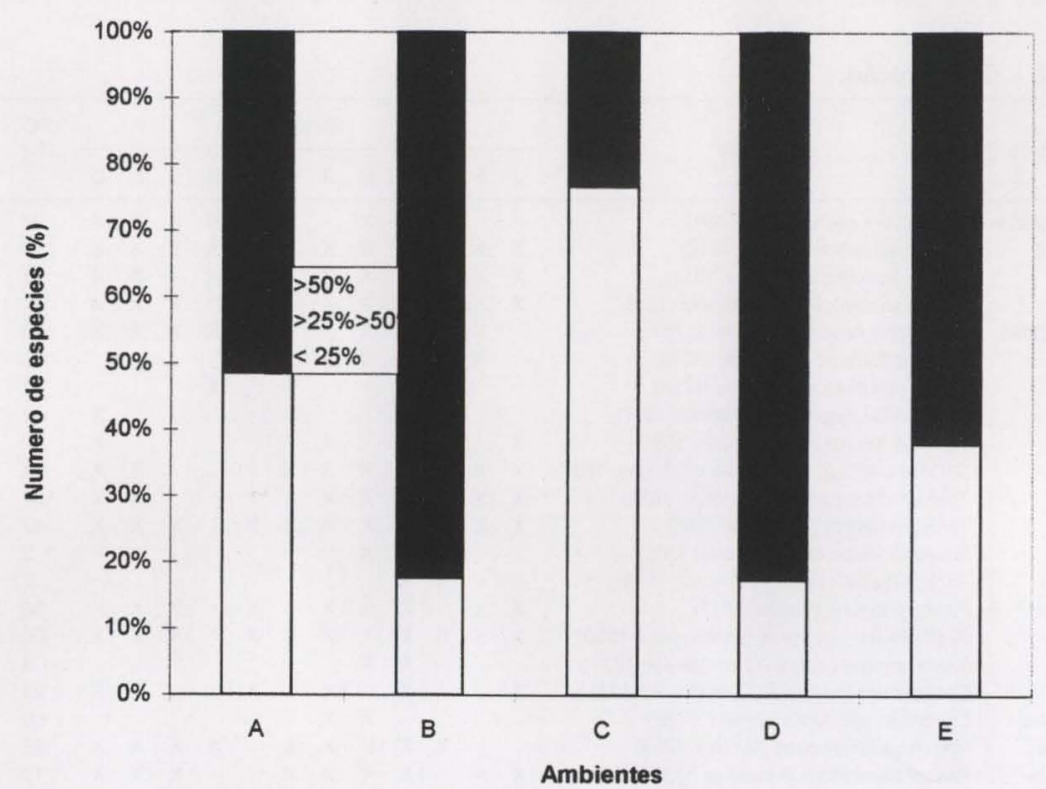

Fig. 2. Número de espécies de aves, expresso em percentagem, com freqüência de ocorrência $<25 \%, 25 \%-50 \%$ e $>50 \%$ nos ambientes A, B, C, D e E.

delas, do que em entornos compostos por gramado com árvores esparsas (ambiente A) e plantação de milho (ambiente C). Entretanto, o fato de a seqüência de ambientes amostrados ter sido sempre a mesma pode ter favorecido o registro de maior número de espécies nos trechos iniciais do transecto, pela maior atividade das aves naquele determinado período da manhã, do que nos trechos finais (Fig. 1).

No ambiente $\mathrm{D}$ foi observada uma modificação na composição das espécies de acordo com a sucessão da vegetação ao redor da floresta. Nos primeiros meses do trabalho havia uma vegetação rasteira em redor deste ambiente, sendo que Vanelus chilensis (Gmelin, 1789) e Crotophaga ani (Linnaeus, 1758) eram freqüentemente registrados ali, mas no momento em que a vegetação foi se transformando em um matagal, essas duas espécies deixaram de ser observadas neste local, embora continuassem a ser registradas nos outros ambientes. Por outro lado, Picumnus temminckii (Temminck, 1825) só passou a ser registrada no ambiente D no momento em que havia se desenvolvido este matagal. Segundo WiLCOVE \& RoBINSON (1990), este acréscimo de algumas espécies de acordo com a sucessão da vegetação é típica de espécies associadas a campos, matagais e florestas jovens.

Apenas 7 espécies mostraram freqüência de ocorrência significativamente maior em um determinado ambiente, quando ocorrem em mais de um (Chi-quadrado): Thraupis sayaca (Linnaeus, 1766) no ambiente A, Turdus leucomelas (Vieillot, 1818) e Basileuterus culicivorus (Lichtenstein, 1830) no ambiente B, Leptopogon amaurocephalus (Tschud, 1846), Troglodytes aedon Vieillot, 1807 e Volatinia jacarina (Linnaeus, 1758) no ambiente D e Dysithamnus mentalis (Temminck, 1823) no ambiente E. A maioria das espécies ocorreu em vários ambientes sem uma ocorrência significativamente maior em um deles (Tab. II). 
Tabela II. Freqüência de ocorrência das espécies de aves nos ambientes ( $A, B, C, D$ e $E)$ e estratos (I - inferior; $M$ - médio; S - superior) do fragmento florestal estudado. $\left(^{*}\right)$ Indica diferença estatística (Chi-quadrado) entre as freqüências de ocorrência da espécie nos ambientes ou nos estratos.

\begin{tabular}{|c|c|c|c|c|c|c|c|c|}
\hline \multirow{2}{*}{ Espécie } & \multicolumn{5}{|c|}{ Ambiente } & \multicolumn{3}{|c|}{ Estrato } \\
\hline & A & B & C & D & $E$ & 1 & M & $\mathrm{s}$ \\
\hline Coragyps atratus & 0,0 & 37,5 & 0,0 & 62,5 & 0,0 & 0,0 & 0,0 & 100,0 \\
\hline Buteo magnirostris & 36,3 & 43,1 & 11,3 & 31,8 & 15,9 & 0,0 & 22,9 & $77,1^{*}$ \\
\hline Vanellus chilensis & 23,0 & 61,5 & 23,0 & 38,4 & 0,0 & 100,0 & 0,0 & 0,0 \\
\hline Columba picazuro & 16,6 & 36,6 & 23,3 & 16,6 & 30,0 & 16,2 & 13,5 & $70,3^{*}$ \\
\hline Columba cayennensis & 21,4 & 35,7 & 21,4 & 21,4 & 21,4 & 47,0 & 0,0 & 53,0 \\
\hline Zenaida auriculata & 31,5 & 39,4 & 28,9 & 44,7 & 0,0 & 40,0 & 29,1 & 30,9 \\
\hline Columbina picui & 0,0 & 100,0 & 0,0 & 0,0 & 0,0 & 0,0 & 50,0 & 50,0 \\
\hline Columbina talpacoti & 22,5 & 40,9 & 18,1 & 40,9 & 22,7 & 34,3 & 50,0 & 15,7 \\
\hline Leptotila verreauxi & 0,0 & 100,0 & 0,0 & 0,0 & 100,0 & 50,0 & 50,0 & 0,0 \\
\hline Leptotila rufaxilla & 0,0 & 0,0 & 0,0 & 0,0 & 100,0 & 50,0 & 50,0 & 0,0 \\
\hline Aratinga leucophthalmus & 0,0 & 0,0 & 0,0 & 50,0 & 75,0 & 0,0 & 0,0 & 100,0 \\
\hline Pyrrhura frontalis & 0,0 & 100,0 & 0,0 & 0,0 & 50,0 & 0,0 & 0,0 & 100,0 \\
\hline Pionus maximiliani & 0,0 & 50,0 & 0,0 & 0,0 & 50,0 & 0,0 & 0,0 & 100,0 \\
\hline Piaya cayana & 11,4 & 34,2 & 8,5 & 57,1 & 25,7 & 12,5 & 47,9 & 39,5 \\
\hline Crotophaga ani & 19,4 & 52,8 & 25,0 & 30,5 & 5,5 & 26,0 & 39,1 & 34,8 \\
\hline Guira guira & 16,6 & 55,5 & 22,2 & 38,8 & 0,0 & 28,0 & 32,0 & 40,0 \\
\hline Nyctidromus albicollis & 0,0 & 0,0 & 100,0 & 50,0 & 0,0 & 100,0 & 0,0 & 0,0 \\
\hline Phaethornis eurynome & 28,5 & 42,8 & 28,5 & 0,0 & 0,0 & 57,1 & 42,9 & 0,0 \\
\hline Phaethornis pretrei & 33,3 & 33,3 & 0,0 & 33,3 & 0,0 & 0,0 & 66,6 & 33,3 \\
\hline Melanotrochilus fuscus & 78,5 & 7,1 & 7,1 & 50,0 & 0,0 & 0,0 & 50,0 & 50,0 \\
\hline Nonnula rubecula & 0,0 & 0,0 & 0,0 & 100,0 & 0,0 & 100,0 & 0,0 & 0,0 \\
\hline Picumnus temminckii & 0,0 & 33,3 & 0,0 & 66,6 & 11,1 & $80,0^{*}$ & 10,0 & 10,0 \\
\hline Veniliornis spilogaster & 0,0 & 20,0 & 0,0 & 60,0 & 40,0 & 0,0 & 100,0 & 0,0 \\
\hline Dryocopus lineatus & 33,3 & 0,0 & 0,0 & 33,3 & 33,3 & 33,3 & 0,0 & 66,6 \\
\hline Furnarius rufus & 0,0 & 100,0 & 0,0 & 0,0 & 0,0 & 50,0 & 0,0 & 50,0 \\
\hline Synallaxis ruficapilla & 23,5 & 41,1 & 8,8 & 35,2 & 20,5 & $86,3^{*}$ & 13,7 & 0,0 \\
\hline Thamnophilus caerulescens & 27,6 & 78,7 & 10,6 & 29,7 & 17,0 & 40,7 & 54,3 & 5,0 \\
\hline Dysithamnus mentalis & 5,2 & 5,2 & 5,2 & 21,0 & $84,2^{*}$ & 47,9 & 52,1 & 0,0 \\
\hline Pyriglena leucoptera & 0,0 & 0,0 & 0,0 & 0,0 & 100,0 & 100,0 & 0,0 & 0,0 \\
\hline Conopophaga lineata & 0,0 & 66,6 & 0,0 & 33,3 & 0,0 & 0,0 & 33,3 & 0,0 \\
\hline Leptopogon amaurocephalus & 0,0 & 23,8 & 0,0 & $95,2^{*}$ & 0,0 & 28,0 & $64,0^{\star}$ & 8,0 \\
\hline Myiornis auricularis & 33,3 & 0,0 & 0,0 & 66,6 & 0,0 & 100,0 & 0,0 & 0,0 \\
\hline Lathrotriccus euleri & 0,0 & 33,3 & 0,0 & 50,0 & 33,3 & 71,4 & 28,6 & 0,0 \\
\hline Colonia colonus & 0,0 & 0,0 & 0,0 & 100,0 & 0,0 & 0,0 & 0,0 & 100,0 \\
\hline Machetornis rixosus & 0,0 & 100,0 & 0,0 & 0,0 & 0,0 & 0,0 & 0,0 & 100,0 \\
\hline Tyrannus savana & 29,4 & 35,2 & 11,7 & 23,5 & 0,0 & 41,2 & 0,0 & 58,8 \\
\hline Tyrannus melancholichus & 0,0 & 100,0 & 0,0 & 0,0 & 0,0 & 0,0 & 0,0 & 100,0 \\
\hline Megarhynchus pitangua & 50,0 & 33,3 & 11,1 & 16,6 & 0,0 & 0,0 & 20,0 & $80,0^{*}$ \\
\hline Myiodynastes maculatus & 21,4 & 35,7 & 0,0 & 28,5 & 28,5 & 0,0 & 25,0 & $75,0^{*}$ \\
\hline Myiozetetes similis & 0,0 & 100,0 & 0,0 & 0,0 & 0,0 & 82,7 & 17,3 & 0,0 \\
\hline Pitangus sulphuratus & 57,6 & 61,5 & 11,5 & 34,6 & 19,2 & 14,7 & 18,7 & $66,6^{*}$ \\
\hline Troglodytes aedon & 0,0 & 16,6 & 33,3 & $100,0^{*}$ & 0,0 & 82,7 & 17,3 & 0,0 \\
\hline Turdus rufiventris & 13,3 & 56,6 & 0,0 & 10,0 & 36,6 & 22,9 & 45,7 & 31,4 \\
\hline Turdus leucomelas & 31,7 & $100,0^{*}$ & 0,0 & 2,4 & 48,7 & 21,6 & 43,0 & 35,4 \\
\hline Turdus amaurochalinus & 16,6 & 72,2 & 0,0 & 25,7 & 16,6 & 20,9 & 50,0 & 29,1 \\
\hline Zonotrichia capensis & 30,7 & 30,7 & 15,3 & 53,8 & 0,0 & 58,8 & 23,5 & 17,7 \\
\hline Volatinia jacarina & 0,0 & 5,2 & 57,8 & $100,0^{*}$ & 0,0 & $65,8^{*}$ & 34,2 & 0,0 \\
\hline Sporophila lineola & 0,0 & 0,0 & 100,0 & 0,0 & 0,0 & 0,0 & 100,0 & 0,0 \\
\hline Sporophila caerulescens & 0,0 & 0,0 & 0,0 & 100,0 & 0,0 & 100,0 & 0,0 & 0,0 \\
\hline Arremon flavirostris & 0,0 & 14,2 & 0,0 & 57,1 & 28,5 & 28,6 & 71,4 & 0,0 \\
\hline Saltator similis & 14,2 & 42,8 & 7,1 & 28,5 & 14,2 & 66,6 & 33,3 & 0,0 \\
\hline Tachyphonus coronatus & 28,5 & 35,7 & 21,4 & 28,5 & 28,8 & 65,0 & 35,0 & 0,0 \\
\hline Thraupis sayaca & $95,8^{*}$ & 41,6 & 0,0 & 0,0 & 0,0 & 0,0 & 24,3 & $75,7^{*}$ \\
\hline Euphonia chlorotica & 0,0 & 0,0 & 100,0 & 0,0 & 0,0 & 0,0 & 0,0 & 100,0 \\
\hline Trichothraupis melanops & 0,0 & 100,0 & 0,0 & 0,0 & 0,0 & 0,0 & 75,0 & 23,0 \\
\hline Parula pitiayumi & 29,4 & 47,0 & 5,8 & 52,9 & 0,0 & 8,8 & 26,0 & $65,2^{*}$ \\
\hline Basileuterus culicivorus & 11,7 & $55,8^{*}$ & 11,7 & 23,5 & 20,5 & 28,5 & 45,2 & 26,3 \\
\hline Basileuterus leucoblepharus & 0,0 & 50,0 & 0,0 & 0,0 & 100,0 & 100,0 & 0,0 & 0,0 \\
\hline Conirostrum speciosum & 7,6 & 23,0 & 15,3 & 76,9 & 0,0 & 18,8 & 43,7 & 37,3 \\
\hline Cyclarhis gujanensis & 0,0 & 100,0 & 0,0 & 33,3 & 0,0 & 0,0 & 25,0 & 75,0 \\
\hline Molothrus bonariensis & 10,5 & 52,6 & 21,0 & 36,8 & 0,0 & 34,7 & 34,7 & 30,6 \\
\hline Passer domesticus & 50,0 & 20,0 & 20,0 & 60,0 & 0,0 & 33,3 & 33,3 & 20,1 \\
\hline
\end{tabular}


O número total de espécies registradas nos estratos foi relativamente semelhante (43 no inferior; 42 no médio e 40 no superior). Entretanto maior percentagem de espécies apresentou freqüência de ocorrência superior a $50 \%$ nos estratos inferior e superior $(41,9 \%$ e $47,5 \%)$ do que no estrato médio (19\%).

Columbina talpacoti (Temminck, 1811) e Piaya cayana (Linnaeus, 1766) foram as espécies que ocorreram em todos os ambientes e estratos em proporções aproximadamente semelhantes podendo ser consideradas as de distribuição espacial mais ampla na área estudada.

Os maiores valores de similaridade (índice de Simpson, Tab. III) foram entre os ambientes A-B e A-D, ambos 93,9. O ambiente A parece ser de transição entre os ambientes B e D; $89,9 \%$ das espécies do ambiente A apresentam freqüência de ocorrência menor que 50\%, mostrando que as aves estão ali de passagem (Fig. 2).

$\mathrm{O}$ menor índice de similaridade foi entre os ambientes C-E. O interior da floresta (ambiente $\mathrm{E}$ ) foi o que apresentou menores índices de similaridade em geral em relação aos outros, todos compostos por borda de floresta. Apenas duas espécies foram exclusivas do ambiente $\mathrm{E}$, embora com baixas freqüências de ocorrência: Leptotila rufaxilla (Richard \& Bernard, 1712) e Pyriglena leucoptera (Vieillot, 1818).

O fato de o ambiente $\mathrm{E}$ (o único constituído apenas por floresta) ser o mais diferenciado e apresentar o menor número de espécies $(80 \%$ delas com freqüência de ocorrência abaixo de $50 \%$ ) em comparação aos outros ambientes demonstra que o efeito de borda parece ser substancial no fragmento florestal estudado.

Tabela III. Índice de similaridade (Simpson) entre os ambientes estudados.

\begin{tabular}{cccccc}
\hline & A & B & C & D & E \\
\hline A & - & - & - & - & - \\
B & 93,9 & - & - & - & - \\
C & 83,3 & 90,0 & - & - & - \\
D & 93,9 & 84,7 & 90,0 & - & - \\
E & 62,0 & 86,2 & 44,8 & 79,3 & - \\
\hline
\end{tabular}

AGRADECIMENTOS. José Marcelo Torezan e Mario Orsi fizeram valiosas sugestões à versão preliminar deste manuscrito. LdA recebe Bolsa Produtividade em Pesquisa do $\mathrm{CNPq}$ (processo 350054/95-9) para estudos sobre aves em fragmentos florestais no norte do Paraná. MRG recebeu Bolsa de Iniciação Científica da Universidade Estadual de Londrina.

\section{REFERÊNCIAS BIBLIOGRÁFICAS}

Aleixo, A. \& J.M.E. Vielliard. 1995. Composição e dinâmica da avifauna da mata de Santa Genebra, Campinas, São Paulo, Brasil. Revta bras. Zool. 12 (3): 493-511.

ANJoS, L. DOs. 1990. Distribuição de aves em uma floresta de araucária da cidade de Curitiba (sul do Brasil). Acta Biol., Curitiba, 19 (1): 51-63.

ANJos, L. DOs \& R. Boçon. 1999. Bird communities in natural forest patches in southern Brazil. The Wilson Bull. 111 (3): 397-414.

ANJos, L. DOS \& S. LAROCA. 1989. Abundância relativa e diversidade em duas comunidades urbanas de aves de Curitiba (sul do Brasil). Arq. Biol. Tecnol., Curitiba, 32: 637-643. 
Anjos, L. Dos; K.L. Schuchmann \& R. BERndT. 1997. Avifaunal composition, species richness, and status in the Tibagi river basin, Paraná State, southern Brazil. Orn. Neotrop. 8 (1): 145-173.

BIERREGAARD JR., R.O. \& T.E. LOVEJOY. 1989. Effects of forest fragmentation on Amazonian understory bird communities. Acta Amazonica 19: 215-241.

Blondel, J. 1991. Birds in biological isolates, p. 45-72. In: C.M. Perrins; J.D. Lebreton \& G.J.M. HIRONS (Eds). Birds population studies: relevance to conservation and management. Oxford, Oxford University Press, 683p.

FormAn, R.T;; A.E. GALLI \& C.F. LECK. 1976. Forest size and avian diversity in New Jersey woodlots with some landuse implications. Oecologia 26: 1-8.

GALLI, A.E.; C.F. LECK \& R.T. FormAN. 1976. Avian distribution patterns within sized forest island in central New Jersey. Auk 93: 356-365.

HolmES, R.T. 1990a. Food resource availability and use in forest bird communities: a comparative view and critique, p. 387-393. In: A. KEAST (Ed.). Biogeography and ecology of forest bird communities. The Hague, SPB Academic Publishing, 410p.

1990b. The structure of a temperate deciduous forest bird communities: variability in time and space, p. 121-139. In: A. KEAST (Ed.). Biogeography and ecology of forest bird communities. The Hague, SPB Academic Publishing, 410p.

MAACK, R. 1981. Geografia Física do Estado do Paraná. Curitiba, 450p.

Meyer de Schauensee, R. 1982. A guide to the birds of South America. Philadelphia, The Academy of Natural Sciences of Philadelphia, 498p.

MOORE, N.W. \& M.D. HoOPER. 1975. On the numbers of birds species in British woods. Biol. Conserv. 8: $239-250$.

Scherer-Neto, P. \& F.C. Straube. 1995. Aves do Paraná: História, lista anotada e bibliografia. Curitiba, Edição dos autores, 79p.

Sick, H. 1997. Ornitologia brasileira. Nova Fronteira, Rio de Janeiro.

SOS Fundação Mata AtLÂNTICA \& INPE. 1990. A evolução dos remanescentes florestais e ecossistemas associados do domínio da mata atlântica: período 1985 a 1990 . São Paulo, mapa impresso por Fundação Valeparaibana de Ensino.

VIELLIARD, J.E.M. \& W.R. SiLVA. 1990. Nova metodologia de levantamento quantitativo da avifauna e primeiros resultados no interior do Estado de São Paulo, Brasil, p. 117-151. In: S. MENDES (Ed.) Anais IV Encontro de Anilhadores de Aves, Recife, Universidade Federal Rural de Pernambuco. Wilcove, D.S. \& S.K. RoBINSON. 1990. The impact of forest fragmentation on bird communities in Eastern North America, p. 319-331. In: A. KEAST (Ed.) Biogeography and ecology of forest bird communities. The Hague, SPB Academic Publishing, 410p.

WILLIS, E.O. 1979. The composition of avian communities in remanescent woodlots in southern Brazil. Paps Avul. Zool., São Paulo, 33 (1): 1-25.

YAHNER, R.H. 1988. Changes in wildlife communities near edge. Conserv. Biol. 2: 333-339.

Recebido em 06.V.1999; aceito em 22.11.2000. 